\title{
Templated growth of II-VI semiconductor optical fiber devices and steps towards infrared fiber lasers
}

\author{
Pier J. A. Sazio ${ }^{* a}$, Justin R. Sparks ${ }^{\mathrm{b}}$, Rongrui He ${ }^{\mathrm{b}}$, Mahesh Krishnamurthi ${ }^{\mathrm{b}}$, Thomas C. Fitzgibbons ${ }^{\mathrm{b}}$, \\ Subhasis Chaudhuri ${ }^{\mathrm{b}}$, Neil F. Baril ${ }^{\mathrm{b}}$, Anna C. Peacock ${ }^{\mathrm{a}}$, Noel Healy ${ }^{\mathrm{a}}$, Venkatraman Gopalan ${ }^{\mathrm{b}}$, \\ John V. Badding \\ ${ }^{a}$ Optoelectronics Research Centre, University of Southampton, SO17 1BJ, UK; \\ ${ }^{b}$ Dept. of Chemistry and Materials Research Institute, Pennsylvania State University, State College, \\ PA 16802, USA
}

\begin{abstract}
ZnSe and other zinc chalcogenide semiconductor materials can be doped with divalent transition metal ions to create a mid-IR laser gain medium with active function in the wavelength range $2-5$ microns and potentially beyond using frequency conversion. As a step towards fiberized laser devices, we have manufactured $\mathrm{ZnSe}$ semiconductor fiber waveguides with low (less than $1 \mathrm{~dB} / \mathrm{cm}$ at $1550 \mathrm{~nm}$ ) optical losses, as well as more complex ternary alloys with $\mathrm{ZnSxSe}(1-\mathrm{x})$ stoichiometry to potentially allow for annular heterostructures with effective and low order mode corecladding waveguiding.
\end{abstract}

Keywords: semiconductor, chalcogenide, optical fibers, waveguides, high pressure chemistry

\section{INTRODUCTION}

Semiconductor waveguide fabrication for photonics is usually performed in a planar geometry. However, over the past decade a new field of semiconductor-based optical fiber devices has emerged. For example, the drawing of soft chalcogenide semiconductor glasses together with low melting point metals allows for meters-long distributed photoconductive detectors ${ }^{1}$. Using high pressure chemical deposition technology jointly developed by our groups at Penn State and ORC Southampton, crystalline elemental semiconductors such as Si or Ge have been chemically deposited at high pressure inside silica capillaries ${ }^{2}$, allowing the optical and electronic properties of these materials to be exploited for applications such as all-fiber optoelectronics ${ }^{3}$. The fiber pores, and thus the wires deposited in them, are exceptionally smooth $^{4}$ with extremely uniform diameter over their entire length. The high-pressure chemical vapor deposition (HPCVD) technique is simple, low cost, and flexible so that it can be modified to fill a range of capillaries with differing core dimensions, while high production rates can be obtained by parallel fabrication of multiple fibers in a single deposition. It can also be extended to fill the large number of micro- and nanoscale pores in microstructured optical fibers (MOFs), thus providing additional geometrical design flexibility to enhance the potential application base of the fiber devices ${ }^{5}$. Semiconductor fibers fabricated via HPCVD in silica pores also retain the inherent characteristics of silica fibers, including their robustness and compatibility with existing optical fiber infrastructure, thus presenting considerable advantages over fibers based on multicomponent soft glasses.

High-performance optoelectronic devices are almost exclusively fabricated from crystalline compound semiconductors owing to their superior light emission efficiency, excellent electronic properties (e.g., high carrier mobility), and large optical nonlinearities. In particular, compound semiconductors can have high second-order nonlinear optical coefficients ${ }^{6}$ not found in either centrosymmetric crystalline unary semiconductors or amorphous semiconductors. These second-order nonlinearities allow for efficient frequency conversion on which devices such as optical parametric oscillators are typically based. Compound semiconductors can also be optically transparent over a wider wavelength range than unary semiconductors, with $\mathrm{ZnSe}$, for example, having excellent optical transmission at wavelengths from 500 to $22000 \mathrm{~nm}$.

*pjas@soton.ac.uk; phone 00442380 593144; fax 00442380 593142; www.orc.soton.ac.uk 
An additional advantage of crystalline compound semiconductors is their ability to host transition metal ions; $\mathrm{C}^{2+}$ doped $\mathrm{ZnSe}$ functions as an efficient gain medium in the 2000 to $3000 \mathrm{~nm}$ region of the infrared spectrum ${ }^{7},{\text { while } \mathrm{Fe}^{2+}}^{\text {doped }}$ $\mathrm{ZnSe}$ is emerging as a medium for longer wavelength applications (4000 to $5000 \mathrm{~nm})^{8}$. The atmospheric transmission windows of $2-5 \mu \mathrm{m}$, coupled with organic and other chemical absorption lines occurring throughout this middle-infrared (mid-IR) wavelength region give rise to a wide variety of medical, scientific, commercial and military applications. Communications, remote sensing, IR countermeasures, laser surgery and non-invasive imaging are just a few of the drivers of high-power solid-state mid-IR laser development. These laser sources must be versatile enough to operate in a variety of temporal modes from continuous wave $(\mathrm{CW})$ all the way to ultrashort pulse while still being widely tunable for wavelength agility. All of this is required at ever increasing power output levels while conforming to size, weight and power consumption limitations under potentially harsh operating environmental conditions. Transition metal-doped zinc selenide lasers operating in the $2-5 \mu \mathrm{m}$ region are thus excellent candidates to help fill these vital roles. In contrast, the varying chemical environment encountered by transition metal dopant ions in oxide and semiconductor glasses generally does not allow for favorable gain properties. A number of analyses have been undertaken in order to investigate this lack of performance, but underlying issues such as unwanted impurities and the strongly covalent nature of the chalcogen glass bonds play a fundamental role. Transition metal cations doped into such glasses ${ }^{9}$ have exhibited long wave fluorescence, yet there has been no demonstration of d-block transition metal lasers. This can be attributed to the fact that TM ions have large emission probabilities compared with RE ions so that the energy level lifetimes are short. Also, in glassy hosts, TM ions can exist in a number of valence states, thus introducing parasitic effects such as reabsorption of laser light, lifetime quenching and trapping of the excitation energy.

\section{RESULTS AND DISCUSSION}

Fiber lasers and amplifiers have a number of advantages over those based on bulk gain media. For example, when the active gain medium is incorporated into the fiber, the light-matter interaction length can be greatly increased, allowing for an optimized gain profile. Fiber lasers are noted for their excellent output beam quality and high coupling efficiency without the need for careful alignment of free space components, allowing for robust, environmentally rugged, lightweight and compact systems design, as well as freedom from unfavorable thermo-optical effects ${ }^{10}$ commonly exhibited by bulk solid state systems. Crystalline ZnSe has much higher thermal conductivity than amorphous soft glasses and melts at much higher temperature $\left(1525^{\circ} \mathrm{C}\right)$. All of these advantages make transition-metal-doped ZnSe fibers attractive for tunable mid-infrared lasers that could operate at far higher output powers than currently possible. As a first step towards such fiber devices, we report centimeters-long high-purity ZnSe compound semiconductor optical fiber waveguides with low optical losses ( $<1 \mathrm{~dB} / \mathrm{cm}$ at $1550 \mathrm{~nm}$ wavelength).

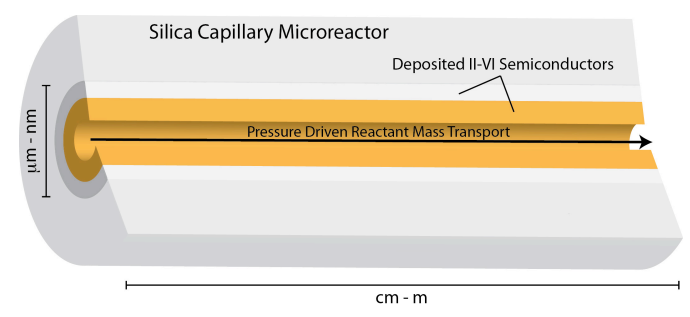

Figure 1. Schematic of the HPCVD process for producing $\mathrm{ZnS}, \mathrm{ZnSe}$, and $\mathrm{ZnS}_{\mathrm{x}} \mathrm{Se}_{(1-\mathrm{x})}$ fibers, where a high pressure chemical precursor mixture (e.g., $(\mathrm{CH} 3)_{2} \mathrm{Zn}$ and $(\mathrm{CH} 3)_{2} \mathrm{Se}$ for $\mathrm{ZnSe}$ ) is configured to flow into a capillary (left). When the capillary is heated, well-developed annular films are deposited that can close down to form nearly void free wires. Unreacted precursors, carrier gas, and reaction byproducts are carried out of the fiber (right). Layers and graded compositions can be realized by changing the precursor chemistry to deposit different semiconductors at different times.

To deposit ZnSe optical fiber cores ${ }^{11}$ (Figure 1), a mixture of Dimethylzinc, Dimethylselenide and hydrogen at a total pressure in the range of 35-70 MPa was configured to flow through silica capillaries heated by a furnace (Figure 1a). The as-deposited material was confirmed to be phase-pure polycrystalline ZnSe in the (isotropic) cubic zinc-blende phase by X-ray diffraction. ZnSe films deposited by conventional techniques typically have root mean square (RMS) 
roughness values of $5-10 \mathrm{~nm}^{12}$. It is therefore reasonable to expect ZnSe tubes deposited via the "outside-in" HPCVD approach to have some interior surface roughness as well. To reduce the area of this interior surface and the optical propagation losses associated with its roughness, the internal diameter of these tubes should be reduced as much as possible. For unary semiconductor waveguides deposited from hydride precursors, we have shown that it is possible to deposit completely void-free waveguides ${ }^{13}$. In such instances, solid wires can be formed because the hydrogen reaction byproduct and helium carrier can diffuse through the silica walls, allowing for continued mass transport and deposition even when the pore is completely blocked downstream. However, the precursor chemistry employed for ZnSe has methane as a reaction byproduct which is too large to diffuse rapidly through the capillary walls and must be exhausted through the interior of the ZnSe tube along with unreacted precursor molecules. When a total pressure of $70 \mathrm{MPa}$ is used, the tube interior diameter can be reduced to as small as $400 \mathrm{~nm}$ forming a nearly void-free core. An interior diameter of this size in waveguides ranging from a few to tens of micrometers in diameter is expected to affect the transmission properties and the optical losses, however, low optical losses on the order of $1 \mathrm{~dB} / \mathrm{cm}$ were still realized. We anticipate that through the use of higher pressures, the interior diameter could be reduced even further or the hole could even be eliminated. As well as simple capillaries, the very high aspect ratio pores in silica microstructured optical fiber (MOF) templates can be deposited with the II-VI materials $\mathrm{ZnS}$ and $\mathrm{ZnS}_{\mathrm{x}} \mathrm{Se}_{(1-\mathrm{x})}$ via $\mathrm{HPCVD}^{14}$. This coating proceeds such that precisely patterned, centimeters-long arrays of wires micrometers in diameter can be formed. $\mathrm{ZnS}_{\mathrm{x}} \mathrm{Se}_{(1-\mathrm{x})}$ is of particular interest because properties such as its refractive index can be continuously tuned with $\mathrm{x}$ over a wide range. Importantly, alloying opens up the possibility to fabricate optical fibers with small core-cladding index contrasts through the deposition of successive layers with varying $\mathrm{x}$, or alternatively, to tune the index contrast in complex 2D photonic crystal structures to shift the positions of the bandgaps. Furthermore, the lattice constant in $\mathrm{ZnS}_{\mathrm{x}} \mathrm{Se}_{(1-\mathrm{x})}$ alloys can also be controlled to allow for continuous tuning of their direct bandgap from 2.7 to $3.6 \mathrm{eV}$, allowing for emission of light from the visible to the near UV.

a)

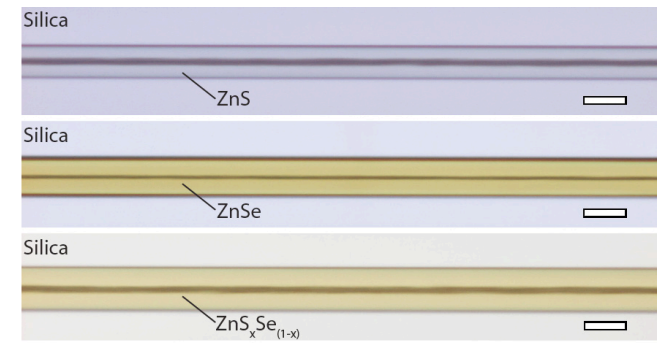

b)

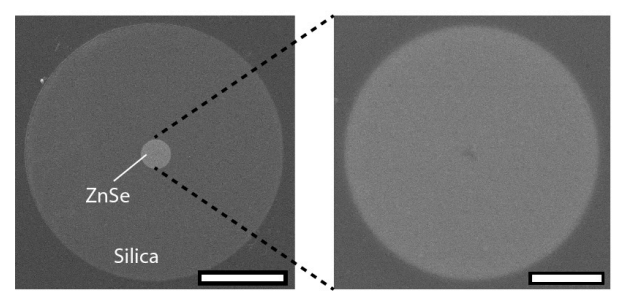

C)

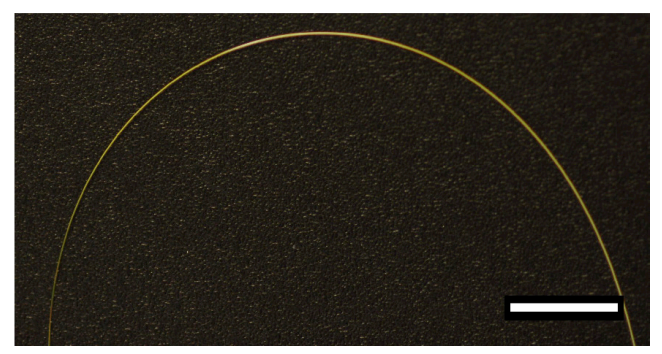

Figure 2. (a) Diascopically illuminated optical microscopy images from the side showing transparent, uniform $\mathrm{ZnS}, \mathrm{ZnSe}$, and $\mathrm{ZnSxSe}(1-\mathrm{x})$ deposited in silica micro-capillaries. Cylindrical lensing effects magnify the core, which nearly completely filled. (b) Overview (left) and higher magnification (right) cross-sectional SEM images of a ZnSe infiltrated capillary showing the $<400 \mathrm{~nm}$ remaining interior pore. (c) Flexible polycrystalline $\mathrm{ZnSe}$ fibre core being bent in silica cladding. Scale bars: a) $20 \mu \mathrm{m}$, b) left: $40 \mu \mathrm{m}$, right: $4 \mu \mathrm{m}$, c) $5 \mathrm{~mm}$ 
By introducting both $(\mathrm{CH} 3)_{2} \mathrm{Se}$ and $(\mathrm{CH} 3)_{2} \mathrm{~S}$ into the reactant flow, solid solutions of $\mathrm{ZnSe}$ and $\mathrm{ZnS}$ can be deposited in well-developed layers (Figure 2a). The kinetic rate constants for decomposition of these two molecules differ, allowing the challenge of controlling the $\mathrm{S}$ to $\mathrm{Se}$ ratio of the $\mathrm{ZnS}_{\mathrm{x}} \mathrm{Se}_{(1-\mathrm{x})}$ solid solution to be met by varying the temperature and precursor ratio. Although these materials can crystallize in either the hexagonal wurtzite structure or the cubic zinc blende structure as the composition is varied, powder X-ray diffraction showed that only the cubic structure was formed in HPCVD when stoichiometries were varied over the entire range from $\mathrm{x}=0$ to $\mathrm{x}=1$. The $\mathrm{S}$ to $\mathrm{Se}$ ratio $(\mathrm{x} / 1-\mathrm{x})$ determined by energy dispersive spectroscopy for materials deposited at different temperatures agreed well with that determined from the measured lattice parameters via Vegard's law. The Raman spectra of the deposited materials are characteristic of $\mathrm{ZnS}_{\mathrm{x}} \mathrm{Se}_{(1-\mathrm{x})}$, exhibiting $\mathrm{TO}_{\mathrm{ZnSe}}, \mathrm{LO}_{\mathrm{ZnSe}}$, and $\mathrm{LO}_{\mathrm{ZnS}}$ phonon modes that shift continuously ${ }^{15}$ with $\mathrm{x}$ (Figure $3)$.
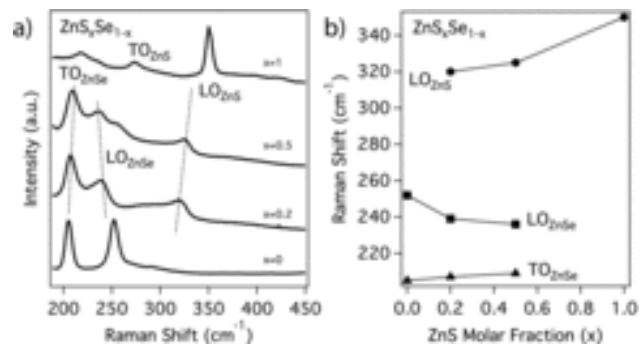

Figure 3. Raman spectroscopy of $\mathrm{ZnS}_{\mathrm{x}} \mathrm{Se}_{(1-\mathrm{x})}$ alloys. The shifts of the $\mathrm{TO}_{\mathrm{ZnSe}}, \mathrm{LO}_{\mathrm{ZnSe}}$, and $\mathrm{LO}_{\mathrm{Zns}}$ phonon modes are characteristic of alloy formation.

The templated deposition of $\mathrm{ZnS}_{\mathrm{x}} \mathrm{Se}_{(1-\mathrm{x})}$ alloys inside microstructured optical fibers can be controlled both compositionally and structurally; for example, the templates can be selectively filled ${ }^{16}$, allowing for accurately patterned and aligned ZnSe wires and tubes with engineered electronic and optical properties. As an example application, the PBG fiber template shown in Figure 4 is filled with $\mathrm{ZnSe}$ in order to realize a novel type of large mode area fiber waveguide, the optical properties of which will be discussed in later sections.
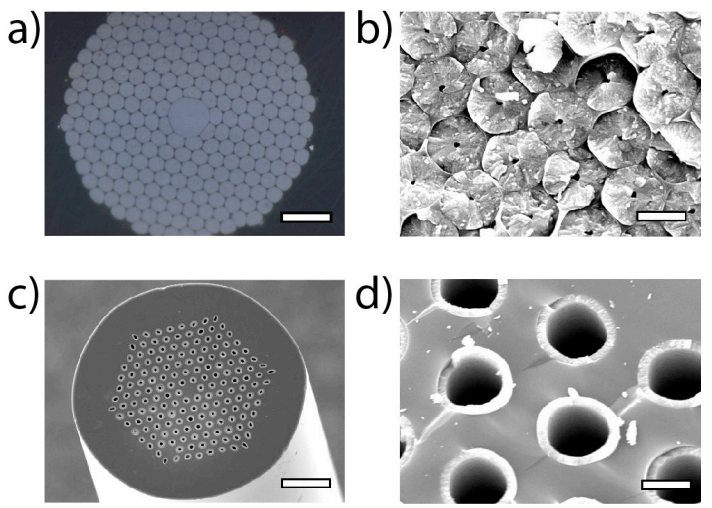

Figure 4. HPCVD in microstructured templates allows for precise positioning of hundreds of II-VI semiconductor wires organized into complex arrays. (a) Cross-sectional optical micrograph of the polished facet of a $\mathrm{ZnSe}$ filled photonic bandgap optical fiber with a high packing density cladding shown in the SEM micrograph (b). (c) Cross-sectional SEM micrograph of a lower packing density array filled with ZnSe tubes, as shown in higher magnification (d). Scale bars: a) 10 $\mu \mathrm{m}$, b) $2.5 \mu \mathrm{m}$, c) $50 \mu \mathrm{m}, \mathrm{d}) 5 \mu \mathrm{m}$.

The near atomically smooth silica pore surfaces are coated with well-developed ZnSe films from the "outside in". Thus it is important to first assess whether the microwires and tubes formed in these pores have comparable outer surface roughness. To do this, we removed an array of $168 \mathrm{ZnSe}$ wires $8 \mu \mathrm{m}$ in diameter deposited in a silica template by etching with HF (Figure 5a). The surface roughness was characterized by means of optical interference profilometry, which uses 
interference between two split beams in a microscope to allow the sub-Ångstrom RMS roughness to be determined over areal dimensions of hundreds of square micrometers. We found that $\mathrm{ZnSe}$ microwires etched out of their template have a RMS surface roughness of $0.1 \mathrm{~nm}$ (Figure 5b), comparable to the surface roughness of the template walls. The capillary templates have extraordinary diameter uniformity along their length as well, such that variations of only tens of nanometers in diameter over lengths of centimeters are possible. Since the $\mathrm{ZnSe}$ and $\mathrm{ZnS}$ microwires conform to the silica template walls, similar uniformity in diameter along their length can be expected. The II-VI semiconductor deposition results in a wire, or more specifically a tube with a very small central void and it is important to assess this inner surface roughness. As the deposition progresses, the flow rate of reactants through the capillary decreases due to the growth of the polycrystalline film in the channel, resulting in a higher residence time of the precursors and the growth of larger grains which eventually forms a blockage and arrests deposition. The roughness of this inner surface will thus be determined by the grain size and shape at this interface. The cross sectional grain structure of the microwire is revealed by both grain boundary etching with $\mathrm{NaOH}$ (Figure 6c) and a TEM image (Figure 6d).

a)

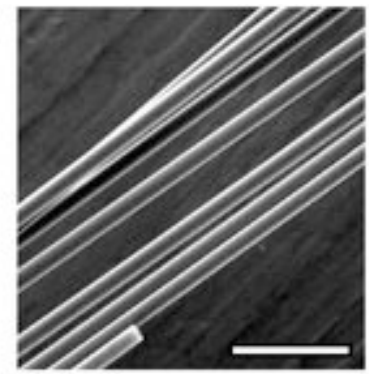

C

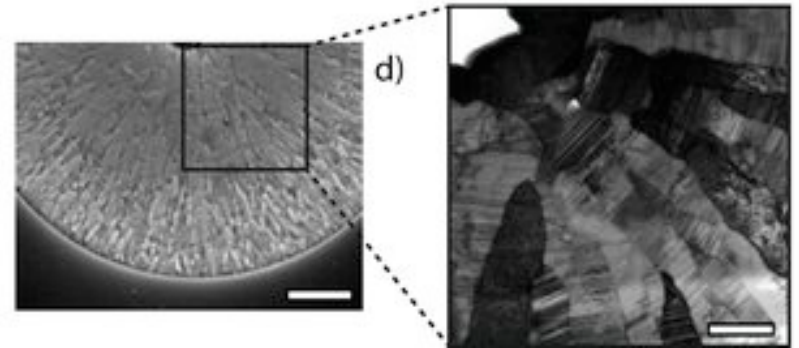

b)

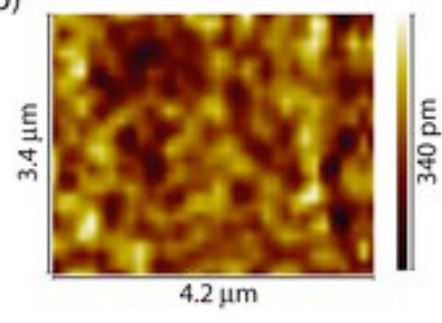

$4.2 \mu \mathrm{m}$

Figure 5. Surface roughness of ZnSe microwires. a) SEM image showing that II-VI microwires can be released from the template by HF etching to result in freestanding microwires. b) Optical interference profiling of a microwire that was removed from the template showing the near atomically smooth outer surface (0.1 nm RMS surface roughness). c) SEM image of $\mathrm{NaOH}$ grain boundary etching to reveal polycrystalline grain structure and size across the cross-section of a microwire, which determines the inner surface roughness of the interior pore. d) TEM image showing large grain structure near the central hole. Scale bars: a) $50 \mu \mathrm{m}$, c) $2 \mu \mathrm{m}$, and d) $500 \mathrm{~nm}$.

No other technique has been reported that can make micro-scale diameter semiconductor wires of crystalline compound semiconductors such as $\mathrm{ZnSe}$ and $\mathrm{ZnS}$ with outer surfaces that are this smooth and uniform in diameter over lengths of centimeters with a circular cross section. Top-down lithographic methods, for example, typically result in semiconductor structures with varying diameters and several nanometers of surface roughness so that achieving $0.1 \mathrm{~nm}$ roughness is exceedingly difficult. Just 1 to $2 \mathrm{~nm}$ of exterior surface roughness on a sub-micrometer-sized waveguide can significantly increase the scattering of visible and near infrared light transported within it ${ }^{17}$. Importantly, these $\mathrm{ZnSe}$ and $\mathrm{ZnS}$ microwires are likely to be useful as very high power laser cavities when suitably doped ${ }^{8}$, thus reducing the losses associated with surface scattering will be critical to achieving high net gains.

In order to test their function as low-loss waveguides, measurements of the transmission loss over the wavelength range of 500 to $2500 \mathrm{~nm}$ of two ZnSe optical fibers fabricated to have core diameters of $15 \mu \mathrm{m}$ and $50 \mu \mathrm{m}$ confirm this expectation (Figure 6). In both cases the losses decrease with increasing wavelength and become as low as $0.5-0.9$ $\mathrm{dB} / \mathrm{cm}$ in the 2000 to $2400 \mathrm{~nm}$ mid-IR region. The loss $\alpha$ as a function of the wavelength can be fitted from 540 to 2400 $\mathrm{nm}$ to a power law with an exponent of $-3.9 \pm 0.2$. This functional dependence is well matched to the $\lambda^{-4}$, where $\lambda$ is the 
wavelength, dependence characteristic of Rayleigh scattering, suggesting that scattering from bulk refractive index inhomogeneities (possibly due to grain boundaries) in the material is the dominant loss mechanism. Thus further engineering of the grain size by optimization of the deposition conditions or thermal or laser annealing may allow for even lower waveguide losses. The intensity profile of $1550 \mathrm{~nm}$ light (Figure 6 inset) at the output of a 15- $\mu \mathrm{m}$-diameter optical fiber is characteristic of multimode behaviour, where the central dip in the intensity is due to the small 400-nmdiameter central pore. As discussed previously, we anticipate that this pore could eventually be eliminated, and we note that further reduction in the overall waveguide diameter to less than $930 \mathrm{~nm}$ would then allow for single-mode behavior at a wavelength of $2400 \mathrm{~nm}$.

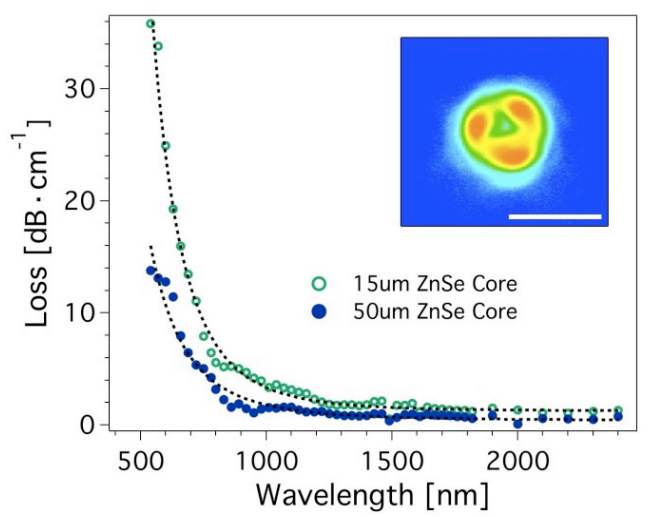

Figure 6. Optical loss of 50 micron (solid/blue) and 15 micron (open/green) diameter $\mathrm{ZnSe}$ core optical fibers determined as a function of wavelength. Dashed lines are a fit to a $\lambda-3.9$ dependence. Inset: Imaged output light at $1550 \mathrm{~nm}$ from a 15micron diameter waveguide. Scale bar is 15 microns.

In general, high refractive index semiconductor $(n=2.45$ for ZnSe at $1550 \mathrm{~nm})$ waveguides with either silica $(n=1.44)$ or air $(n=1)$ cladding have a large index contrast and thus are highly multimode. Realizing micrometer-sized semiconductor waveguides with more desirable low order mode or single mode behavior is still an ongoing challenge in photonics that requires proper refractive index grading. As previously mentioned, a key advantage of HPCVD in MOFs is the ability to deposit materials in complex geometries to modify the guiding properties of light in the semiconductor wires. In particular, the structure shown in Figure $4 \mathrm{a}$ is the first demonstration of a $\mathrm{ZnSe} \mathrm{MOF}$, similar to the silicon MOF that was characterized ${ }^{5}$, which was shown to have a number of analogous properties to more standard air-silica MOFs. The fiber was fabricated by filling the air holes of a hollow core silica photonic bandgap fiber with ZnSe via HPCVD. The resulting structure has a $9 \mu \mathrm{m} \mathrm{ZnSe}$ core with a cladding of $3 \mu \mathrm{m} \mathrm{ZnSe} \mathrm{wires} \mathrm{with} \approx 150 \mathrm{~nm}$ silica struts separating each wire. Compared to the step index fibers above, the ZnSe MOF has a cladding with a higher effective index than pure silica so that a reduced number of core modes can be selectively coupled. This ZnSe fiber is effectively dual mode over a wide wavelength range (Figure 7), though with further optimization of the MOF template and deposited material it should be possible to design a $\mathrm{ZnSe}$ MOF for effective single mode operation. Transmission measurements in this micro- structured ZnSe fiber at $1550 \mathrm{~nm}$ show it has a loss of $1.9 \mathrm{~dB} / \mathrm{cm}$. This slightly higher loss value than in a step index $\mathrm{ZnSe}$ optical fiber is likely to be due to the enhanced interaction of the core guided light with the high index microstructured cladding. 
a)

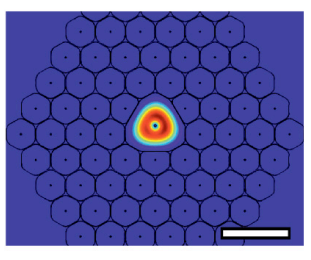

C)

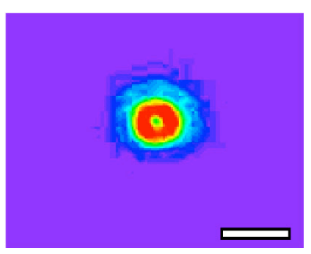

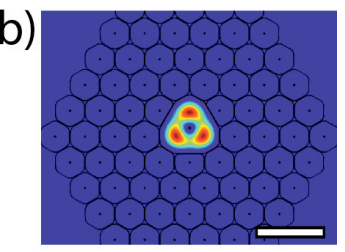

d)

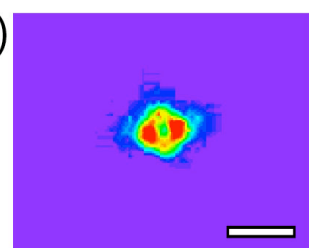

Figure 7. Finite element simulations of the ZnSe MOF shown in Fig.4 where (a) is the fundamental mode and (b) is the second order mode calculated at $1550 \mathrm{~nm}$. Experimental waveguiding experiments at $1550 \mathrm{~nm}$ show that the fundamental mode (c) and second order mode (d) can be isolated. Scale bars: $10 \mu \mathrm{m}$

\section{SUMMARY}

The integration of crystalline ZnSe into optical fibers to form low-loss waveguides that can now exploit the superior properties of crystalline compound semiconductors for the future fabrication of photonic devices, such as fiber amplifiers and lasers, as well as for nonlinear optical processing was demonstrated. Extending this technology for the deposition of ordered arrays of semiconductor structures in MOF templates is straightforward and allows for large-mode-area guidance, as previously demonstrated for silicon in $\mathrm{MOFs}^{5}$. We note that the approach to fabricating ZnSe core optical fibers via HPCVD reported here should be applicable to a broad range of compound semiconductors via assessment and development of suitable precursor chemistries. The range of high-performance electronic and optoelectronic functions that could then be integrated into optical fiber devices extends far beyond what is currently possible with amorphous materials and crystalline unary semiconductors.

\section{ACKNOWLEDGEMENTS}

The authors acknowledge EPSRC (EP/I035307/1), NSF (DMR-0806860 and DMR-1107894), The Pennsylvania State University Materials Research Institute Nano Fabrication Network and the National Science Foundation (NSF) (Cooperative Agreement no. 0335765), National Nanotechnology Infrastructure Network, with Cornell University and the Penn State Materials Research Science and Engineering Center (NSF DMR-0820404) for financial support. We also acknowledge Air Force Contract \#FA8650-10-C-1902 and valuable discussions with Ken Schepler and Patrick Berry.

\section{REFERENCES}

[1] Bayindir, M., Sorin, F., Abouraddy A. F., Viens, J., Hart, S. D., Joannopoulos, J. D. and Fink, Y., "Metalinsulator semiconductor optoelectronic fibres", Nature 431, 826-829 (2004).

[2] Sazio, P. J. A., Amezcua-Correa, A., Finlayson, C. E., Hayes, J. R., Scheidemantel, T. J., Baril, N. F., Jackson, B. R., Won, D. J., Zhang, F., Margine, E. R., Gopalan, V., Crespi, V. H. and Badding, J. V., "Microstructured optical fibers as high pressure microfluidic reactors", Science 311, 1583-1586 (2006).

[3] He, R., Sazio, P. J. A., Peacock, A. C., Healy, N., Sparks, J. R., Krishnamurthi, M., Gopalan, V. and Badding, J.V., "Integration of gigahertz-bandwidth semiconductor devices inside microstructured optical fibres", Nature Photonics 6, 174-179 (2012).

[4] Roberts, P. J., Couny, F., Sabert, H., Mangan, B. J., Williams, D. P., Farr, L., Mason, M. W., Tomlinson, A., Birks, T. A., Knight, J. C. and Russell, P. S. J., "Ultimate low loss of hollow-core photonic crystal fibres", Optics Express 13, 236-244 (2005). 
[5] Healy, N., Sparks, J. R., Petrovich, M. N., Sazio, P. J. A., Badding, J. V and Peacock, A. C., "Large mode area silicon microstructured fiber with robust dual mode guidance", Optics Express 17, 18076-18082 (2009).

[6] Wagner, H. P., Kuhnelt, M., Langbein, W. and Hvam, J. M., "Dispersion of the second-order nonlinear susceptibility in ZnTe, ZnSe, and ZnS", Physical Review B 58, 10494-10501 (1998).

[7] Sorokina, I. T., "Cr2+ doped II-VI materials for lasers and nonlinear optics”, Optical Materials 26, 395-412 (2004).

[8] Mirov, S., Fedorov, V., Moskalev, I., Martyshkin, D. and Kim, C., "Progress in Cr2+ and Fe2+ doped mid-IR laser materials", Laser Photonics Review 4, 21-41 (2010).

[9] Hughes, M. A., Curry, R. J. and Hewak, D. W., "Spectroscopy of Ti-doped GLS glass", Journal of the Optical Society of America B 25, 1458-1465 (2008).

[10]Knight, J. C., "Photonic crystal fibers and fiber lasers", Journal of the Optical Society of America B 24, 16611668 (2007).

[11]Sparks, J. R., He, R., Healy, N., Krishnamurthi, M., Peacock, A. C., Sazio, P. J. A., Gopalan, V. and Badding, J. V., "Zinc selenide optical fibers", Advanced Materials 23, 1647-1651 (2011).

[12] Thiandoume, C., Angermeier, D. and Gorochov, O., "Modeling and Process Optimization of ZnSe and ZnS Epitaxial Growth in a Vertical Metallorganic Vapor Phase Epitaxy Reactor", Journal of the Electrochemical Society 145, 2887-2891 (1998).

[13] Baril, N. F., Keshavarzi, B., Sparks, J. R., Krishnamurthi, M., Temnykh, I., Sazio, P. J. A., Peacock, A. C., Borhan, A., Gopalan, V. and Badding, J. V., "High-Pressure Chemical Deposition for Void-Free Filling of Extreme Aspect Ratio Templates”, Advanced Materials 22, 4605-4611 (2010).

[14] Sparks, J. R., He, R., Healy, N., Chaudhuri, S., Fitzgibbons, T. C., Peacock, A. C., Sazio, P. J. A. and Badding, J. V., "Conformal coating by high pressure chemical deposition for patterned microwires of II-VI semiconductors", Advanced Functional Materials 23, 1647-1654 (2013).

[15]Brafman, O., Chang, I. F., Lengyel, G., Mitra, S. S. and Carnall, E., "Optical phonons in ZnSxSe(1-x) mixed crystals" Physical Review Letters 19, 1120-1123 (1967).

[16]Sparks, J. R., Esbenshade, J. L., He, R., Healy, N., Day, T. D., Keefer, D. W., Sazio, P. J. A., Peacock, A. C. and Badding, J. V., "Selective semiconductor filling of microstructured optical fibers" Journal of Lightwave Technology 29, 2005-2008 (2011).

[17]Lee, K. K., Lim, D. R., Luan, H., Agarwal, A., Foresi, J. and Kimerling L. C., "Effect of size and roughness on light transmission in a Si/SiO2 waveguide: Experiments and model", Applied Physics Letters 77, 1617-1619 (2000). 